\title{
Functional Interactions between Mammalian Respiratory Rhythmogenic and Premotor Circuitry
}

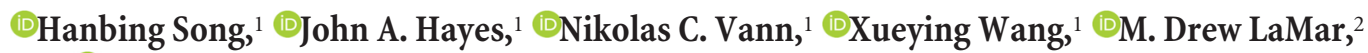 \\ and $\mathbb{C}^{\text {Christopher A. Del Negro }}{ }^{1}$ \\ Departments of ${ }^{1}$ Applied Science and ${ }^{2}$ Biology, College of William and Mary, Williamsburg, Virginia 23187
}

Breathing in mammals depends on rhythms that originate from the preBötzinger complex (preBötC) of the ventral medulla and a network of brainstem and spinal premotor neurons. The rhythm-generating core of the preBötC, as well as some premotor circuits, consist of interneurons derived from Dbx1-expressing precursors (Dbx1 neurons), but the structure and function of these networks remain incompletely understood. We previously developed a cell-specific detection and laser ablation system to interrogate respiratory network structure and function in a slice model of breathing that retains the preBötC, the respiratory-related hypoglossal (XII) motor nucleus and XII premotor circuits. In spontaneously rhythmic slices, cumulative ablation of Dbx1 preBötC neurons decreased XII motor output by $\sim 50 \%$ after $\sim 15$ cell deletions, and then decelerated and terminated rhythmic function altogether as the tally increased to $\sim 85$ neurons. In contrast, cumulatively deleting Dbx1 XII premotor neurons decreased motor output monotonically but did not affect frequency nor stop XII output regardless of the ablation tally. Here, we couple an existing preBötC model with a premotor population in several topological configurations to investigate which one may replicate the laser ablation experiments best. If the XII premotor population is a "small-world" network (rich in local connections with sparse long-range connections among constituent premotor neurons) and connected with the preBötC such that the total number of incoming synapses remains fixed, then the in silico system successfully replicates the in vitro laser ablation experiments. This study proposes a feasible configuration for circuits consisting of Dbx1-derived interneurons that generate inspiratory rhythm and motor pattern.

Key words: central pattern generator; pre-Bötzinger complex; respiration

\section{Significance Statement}

To produce a breathing-related motor pattern, a brainstem core oscillator circuit projects to a population of premotor interneurons, but the assemblage of this network remains incompletely understood. Here we applied network modeling and numerical simulation to discover respiratory circuit configurations that successfully replicate photonic cell ablation experiments targeting either the core oscillator or premotor network, respectively. If premotor neurons are interconnected in a so-called "small-world" network with a fixed number of incoming synapses balanced between premotor and rhythmogenic neurons, then our simulations match their experimental benchmarks. These results provide a framework of experimentally testable predictions regarding the rudimentary structure and function of respiratory rhythm- and pattern-generating circuits in the brainstem of mammals.

\section{Introduction}

Inspiratory breathing movements emanate from neural activity in the preBötzinger complex (preBötC) (Smith et al., 1991; Feld-

Received Jan. 27, 2016; revised May 27, 2016; accepted May 27, 2016.

Author contributions: H.S., J.A.H., X.W., and C.A.D.N. designed research; H.S., J.A.H., N.C.V., and M.D.L. performed research; H.S., M.D.L., and C.A.D.N. analyzed data; H.S., J.A.H., M.D.L., and C.A.D.N. wrote the paper.

This work was supported by National Institutes of Health Grant HL104127 to C.A.D.N.

The authors declare no competing financial interests.

Correspondence should be addressed to Dr. Christopher A. Del Negro, College of William and Mary, Department of Applied Science, McGlothlin-Street Hall, Williamsburg, VA 23187-8795. E-mail: cadeln@wm.edu.

X. Wang's present address: Department of Molecular and Cellular Biology, Harvard University, Cambridge, Massachusetts 02138. man and Del Negro, 2006; Feldman et al., 2013; Moore et al., 2013) as well as a network of premotor neurons that transforms inspiratory rhythm into a coordinated set of motor commands serving ventilation. Because our knowledge of the preBötC presently exceeds that of the premotor circuits, we used experiments and modeling to advance understanding of how brainstem premotor neurons interconnect with the preBötC and influence inspiratory-related motor patterns.

Interneurons whose progenitors express the homeodomain transcription factor Dbx1 (hereafter referred to as Dbx1 neurons) 
may comprise the rhythmogenic core of the preBötC (Bouvier et al., 2010; Gray et al., 2010; Picardo et al., 2013). Rhythms that originate in the preBötC drive a complement of respiratory muscles, including the tongue protractor (genioglossus) that helps maintain airway patency during breathing behavior. Hypoglossal (XII) motoneurons in the dorsomedial medulla innervate these protractor muscles. XII premotor neurons, which convey inspiratory-related drive to XII motoneurons, have been identified in the intermediate reticular formation intercalated between the preBötC and the XII motor nucleus (Ono et al., 1994; Woch et al., 2000; Peever et al., 2002; Koizumi et al., 2008; Fregosi et al., 2011). Interestingly, Dbxl neurons also situated in the intermediate reticular formation adjacent to preBötC, constitute a significant set of XII premotor neurons (Wang et al., 2014; Revill et al., 2015).

Positing that Dbxl preBötC neurons largely contribute to rhythmogenesis and Dbx1 reticular neurons primarily contribute to premotor drive transmission, we tested those roles experimentally in a rhythmically active slice model of breathing (Funk and Greer, 2013). We used photonics to cumulatively destroy Dbx1 preBötC neurons while monitoring rhythmic motor output in real time. After $\sim 15$ ablations, the magnitude of inspiratory motor output measured from the XII nerve root decreased by half; further ablations slowed spontaneous rhythmic frequency, which ceased after an ablation tally of $85 \pm 20$ (Wang et al., 2014). Later we adapted the technique to destroy Dbx1 neurons from the intermediate reticular formation, which decreased the magnitude of XII motor output linearly without affecting the frequency of the respiratory rhythm (Revill et al., 2015).

The observations that ablations in the preBötC exerted an immediate and profound amplitude effect on XII motor output, whereas cumulative ablations in the reticular formation attenuated XII motor output in a graded manner, were nonintuitive and unexpected results. Therefore, we aimed to construct a model XII premotor network (i.e., a simulated intermediate reticular formation) to connect to an established model preBötC (Song et al., 2015) such that, when cumulatively deleting neurons from either population, the frequency and amplitude of the fictive nerve output would match the experimental results in the targeted laser ablation experiments summarized above.

This study provides a feasible blueprint for the assembly of a rudimentary rhythm and pattern-generating neural circuit that improves our understanding of how Dbxl-derived interneurons of the lower medulla generate and regulate breathing behavior.

\section{Materials and Methods}

\section{Rubin-Hayes preBötC neuron model}

Each preBötC neuron is a Rubin-Hayes model (Rubin et al., 2009; Dunmyre et al., 2011), featuring Hodgkin-Huxley-like spiking currents with four additional currents: calcium-activated nonspecific cation current $\left(I_{\text {CAN }}\right)$ (Crowder et al., 2007; Pace et al., 2007; Mironov, 2008, 2013; Pace and Del Negro, 2008; Mironov and Skorova, 2011), excitatory synaptic current mediated by AMPA receptors $\left(I_{\text {syn }}\right)$ (Funk et al., 1993; Ge and Feldman, 1998), persistent sodium current $\left(I_{\mathrm{Na}-\mathrm{P}}\right)$ (Del Negro et al., 2002; Ptak et al., 2005; Koizumi and Smith, 2008), and Na/K ATPase pump current $\left(I_{\text {pump }}\right)$ (Del Negro et al., 2009; Krey et al., 2010). The Rubin-Hayes model is in the public domain (http://senselab.med.yale. edu/modeldb/ShowModel.asp? model $=125649)$.

The current-balance equation takes the following form:

$$
\begin{aligned}
C \frac{d V}{d t}=-I_{\text {leak }}(V) & -I_{N a}(V, m, h)-I_{K}(V, n)-I_{C A N}(V, C a) \\
& -I_{N a P}\left(V, h_{N a-P}\right)-I_{s y n}\left(V, s_{1} \ldots s_{N}\right)-I_{\text {pump }}(N a)
\end{aligned}
$$

where

$$
\begin{gathered}
\frac{d x}{d t}=\left(x_{\infty}(V)-x\right) / T_{x}(V) \\
\frac{d s}{d t}=\left((1-s) s_{\infty}(V)-k_{s} s\right) / T_{s} \\
\frac{d C a}{d t}=\varepsilon\left(\sum_{i=1}^{N} s_{i} \cdot k_{s y n C a}-k_{C a}\left(C a-C a_{\infty}\right)\right)
\end{gathered}
$$

and

$$
\frac{d N a}{d t}=\alpha\left(-I_{C A N}(V, C a)-I_{\text {pump }}(N a)\right)
$$

describe the evolution of the state variables, for each $x$ in $\left\{m, h, n, h_{\mathrm{Na}-\mathrm{P}}\right\}$.

Whole-cell currents are described with chord-conductance equations, in some cases modified for $\mathrm{Ca}^{2+}$ or $\mathrm{Na}^{+}$gating ( $\mathrm{Li}$ and Rinzel, 1994) as follows:

$$
\begin{gathered}
I_{\text {leak }}(V)=g_{\text {leak }}\left(V-E_{L}\right) \\
I_{N a}(V, m, h)=g_{N a} m^{3} h\left(V-E_{N a}\right) \\
I_{N a-P}\left(V, h_{N a-P}\right)=g_{N a-P} m_{N a-P_{\infty}} h_{N a-P}\left(V-E_{N a}\right) \\
I_{K}(V, n)=g_{K} n^{4}\left(V-E_{K}\right) \\
I_{C A N}(V, C a)=g_{C A N}\left(V-E_{C A N}\right) /\left(1+\exp \left(\left(C a-k_{C A N}\right) / \sigma_{C A N}\right)\right) \\
I_{s y n}\left(V, s_{1} \ldots s_{N}\right)=g_{s y n} \sum_{i=1}^{N} s_{i}\left(V-E_{s y n}\right)
\end{gathered}
$$

where $N$ is the number of presynaptic neurons, $\left\{s_{1} \ldots s_{N}\right\}$ reflects presynaptic $s$ variables, and

$$
I_{\text {pump }}(N a)=r_{\text {pump }}\left(\phi(N a)-\phi\left(N a_{\infty}\right)\right) .
$$

The remaining functions, including those for voltage-dependent channel gating, are as follows:

$$
\begin{gathered}
x_{\infty}(V)=1 /\left(1+\exp \left(\frac{V-\theta_{x}}{\sigma_{x}}\right)\right) \\
T_{x}(V)=T_{x_{\max }} / \cosh \left(\frac{V-\theta_{x}}{2 \sigma_{x}}\right) \\
\phi(N a)=N a^{3} /\left(N a^{3}+k_{N a}^{3}\right) .
\end{gathered}
$$

Model parameters are set to the following values for preBötC neurons: $C=45 p F, g_{\text {leak }}=3 \pm 0.78 n S, E_{L}=-61.46 m V, g_{N a}=150 n S$, $E_{N a}=65 m V, g_{N a-P}=1 n S, g_{K}=30 n S, E_{K}=-75 m V, g_{C A N}=4 \pm 0.75 n S$, $E_{C A N}=0 \mathrm{mV}, g_{s y n}=3.25 \mathrm{nS}, E_{s y n}=0 \mathrm{mV}, \theta_{m}=-36 \mathrm{mV}, \sigma_{m}=-8.5 \mathrm{mV}$, $T_{m_{\max }}=1 \mathrm{~ms}, \theta_{h}=-30 \mathrm{mV}, \sigma_{h}=5 \mathrm{mV}, T_{h_{\max }}=15 \mathrm{~ms}, \theta_{n}=-30 \mathrm{mV}$, $\sigma_{n}=-5 \quad m V, T_{n_{\max }}=30 \quad m s, \theta_{s}=15 \quad m V, \sigma_{s}=-3 \quad m V, \mathrm{~T}_{s}=15 \quad \mathrm{~ms}$, $\theta_{m_{N a-P}}=-40 \mathrm{mV}, \sigma_{m_{N a-P}}=-6 \mathrm{mV}, \theta_{h_{N a-P}}=-48 \mathrm{mV}, \sigma_{h_{N a-P}}=6 \mathrm{mV}$, $T_{\mathrm{Na}-P}=1000 \mathrm{~ms}, k_{\mathrm{Ca}} \stackrel{\mathrm{Na}=}{=} 22.5 \mathrm{~ms}^{-1}, \theta_{\mathrm{CAN}}=0.9 \mu \mathrm{M}, \sigma_{\mathrm{CAN}}=-0.05 \mu \mathrm{M}$, $\mathrm{k}_{\mathrm{s}}=1, k_{\text {synCa }}=1200 \mu M \cdot \mathrm{ms}^{-1}, \mathrm{r}_{\text {pump }}=200 \mathrm{pA}, \mathrm{k}_{\mathrm{Na}}=10 \mathrm{mM}$, $C a_{\infty}=0.05 \mu M, N a_{\infty}=5 \mathrm{mM}, \varepsilon=0.0007, \alpha=6.6 \times 10^{-5} \mathrm{mM} \cdot \mathrm{pA}^{-1} \cdot \mathrm{ms}^{-1}$.

We modeled XII premotor neurons using the Rubin-Hayes formulation with a few parameters adjusted to fit measurements of respiratory premotor neurons (Koizumi et al., 2008):

$E_{L}=-65 \pm 1.8 m V, g_{\text {leak }}=1.23 \times(3 \pm 0.78) n S$,

$$
g_{\mathrm{Na}-\mathrm{P}}=0.45 \mathrm{nS} .
$$

The synaptic gating variable $s$, which was coupled to the ordinary differential equation for $\mathrm{Ca}^{2+}$, represents both ionotropic and metabotropic glutamatergic receptor activation. 
Network model consisting of the preBötC and intermediate reticular formation

We previously modeled the preBötC as a directed Erdős-Rényi random graph (Newman et al., 2001). The model preBötC with $n=250$ interneurons and interconnection probability $p=0.1625$ produces a rhythm with a realistic mean cycle period (Wang et al., 2014).

Premotor interneurons that project to inspiratory-related XII motoneurons have been identified in the intermediate reticular formation, which is intercalated between the preBötC and the XII nucleus (Dobbins and Feldman, 1994; Ono et al., 1994; Peever et al., 2002; Chamberlin et al., 2007; Koizumi et al., 2008; Volgin et al., 2008; Stanek et al., 2014). Because the intermediate reticular formation contains oral-motor as well as respiratory interneurons, the total number of XII premotor neurons dedicated to respiration is unknown. Koizumi et al. (2008) functionally identified 146 XII premotor neurons in rhythmically active slice preparations from neonatal rodents in vitro: 136 located in the intermediate reticular formation and 10 within the dorsal part of the preBötC. These functionally identified premotor interneurons expressed a variety of transmitter phenotypes.

Our model network selectively incorporates glutamatergic Dbx1derived premotor neurons in the intermediate reticular formation and the dorsal preBötC (Wang et al., 2014; Revill et al., 2015). We fixed the Dbx1 reticular premotor population size at $n=100$, which is based on the Koizumi et al. (2008) estimate but slightly reduced to account for functionally identified inspiratory XII premotor neurons that are not glutamatergic. Reticular premotor neurons receive synaptic input from the preBötC but do not project back to it (Koshiya and Smith, 1999; Koizumi et al., 2008). This coupling arrangement reflects the orthodromic mode of transmission from preBötC to premotor neurons to genioglossus XII motoneurons. All synaptic connections in this study are excitatory.

To represent the fraction of Dbx1 XII premotor neurons within the preBötC (Wang et al., 2014), we randomly selected 50 constituent neurons that interconnect locally in the preBötC but, furthermore, contribute directly to network output (thus modeling a projection to the XII nucleus). Fictive nerve output was quantified as a running-time spike histogram from the raster plot of spike activity in all 100 reticular premotor neurons and 50 preBötC neurons (i.e., $20 \%$ of the preBötC population) that project directly to the XII nucleus (Wang et al., 2014). The amplitude of this ensemble network rhythm represents fictive XII nerve output (i.e., it mimics experimental recordings of XII motor nerve roots in vitro) (Smith et al., 1991; Funk and Greer, 2013) and thus is depicted as distinct sites of projection in Figure $1 A$.

Figure $1 A$ provides a diagram of the model system. Black elements, including the population size of the preBötC and reticular premotor population, remain fixed, whereas magenta elements were adjusted, such as (1) the specific number of preBötC neurons that project to the reticular formation, (2) the connectivity from preBötC to reticular premotor neurons, and (3) the connectivity among reticular premotor interneurons.

The fraction of preBötC neurons that were candidates for projection to the reticular premotor neurons (i.e., allowable fraction) was varied from 0.2 to 0.45 with a step size of 0.025 . Whether a preBötC neuron in this reticular-projecting subset actually synapsed onto each individual XII premotor neuron was further governed by a connection probability that varied from 0.15 to 0.4 with a step size of 0.025 . For each parameter pair, we performed 10 simulations (i.e., 10 unique network realizations). Qualitative network behavior appears to depend inversely on the allowable fraction of projected preBötC neurons and the connection probability. If the allowable fraction equals or exceeds 0.35 and the connection probability exceeds 0.2 , then the XII premotor population fully synchronizes with the preBötC rhythm (Fig. 1B).

Connectivity among XII premotor neurons is unknown, so we examined three canonical network structures: Erdős-Rényi (random), scalefree, and small-world (Watts and Strogatz, 1998; Barabasi and Albert, 1999; Newman et al., 2001). Each constituent neuron is a vertex in the network, and its connections are dubbed edges (or links). Figure $1 \mathrm{~A}$ provides a schematic of connectivity but does not accurately depict the topology of vertices and edges.
A
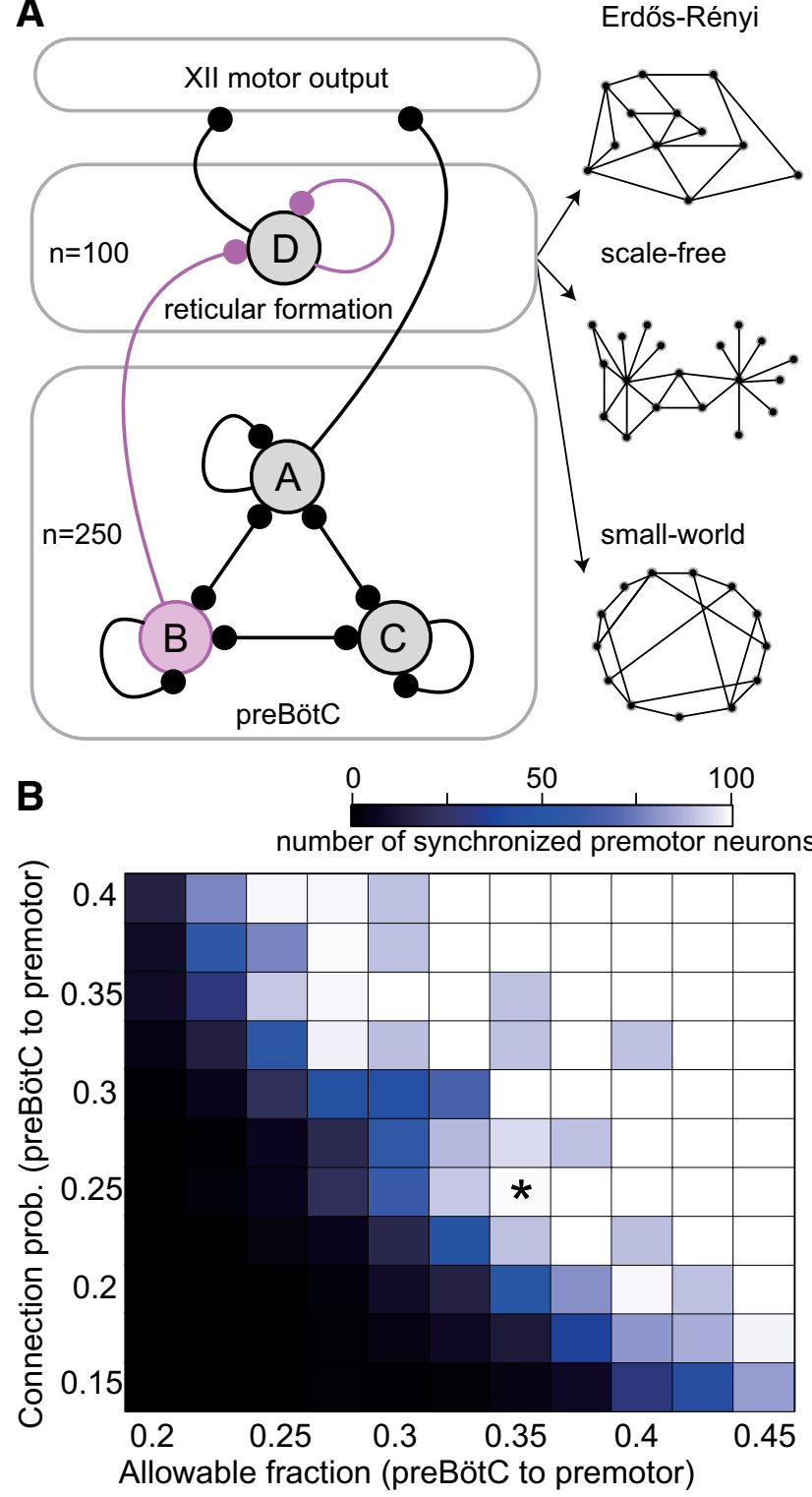

Figure 1. Diagram of the preBötC, intermediate reticular formation, and XII nucleus, which generate inspiratory-related rhythm and motor output, respectively. $A$, The preBötC network (bottom lozenge) consists of 250 neurons. Some preBötC neurons (set B) are allowed to connect to the reticular formation premotor system (middle lozenge), 50 preBötC neurons (set A) directly connect to model XII nucleus to influence fictive nerve output. The rest of the preBötC neurons only connect within the preBötC (set C). All preBötC neurons (sets $A-C$ ) are allowed to connect to each other with a fixed connection probability. The reticular premotor system consists of 100 neurons (set D) that all project to the model XII nucleus. Circles represent neuron populations. Lines with round terminations indicate directed synaptic projections. Synaptic projections among neurons within the same population are indicated by a recurrent connection (e.g., the line projecting from set $A$ back into set A). Interconnection between the preBötC and the reticular premotor system is either Erdós-Rényi (random) or obeys an antipreferential attachment rule. The synaptic interconnection for constituent neurons of the reticular premotor system is either Erdős-Rényi, scale-free, or small-world (set D). Specific connection topologies are not accurately represented in this schematic, which rather shows a basic schematic of the whole system. We also show three diagrams representing three characteristic networks: ErdősRényi ( $\boldsymbol{A}$, right top), scale-free ( $\boldsymbol{A}$, right middle), and small-world network ( $\boldsymbol{A}$, right bottom), respectively. $\boldsymbol{B}$, Interconnection between model preBötC and reticular premotor networks as a function of the allowable fraction (i.e., the fixed proportion of preBötC neurons that may connect to reticular premotor neurons) and probability that they do so. Blocks show the number of synchronized premotor neurons according to the colorimetric scale (top) for one 30 s simulation. No simulated deletions were performed. *Indicates the parameter pair $(0.36,0.25)$ that ensures full synchronization of constituent premotor neurons. 
Erdös-Rényi. Each vertex is connected with equal probability $p$ to any of the other $n-1$ vertices in the network (Newman et al., 2001). Figure $1 A$ (right, top) shows a diagram of an Erdős-Rényi network.

Scale free. The degree (number of connections a vertex possesses, in or out) distribution for all vertices follows a power law. Scale-free networks feature hubs (i.e., vertices that are very highly connected compared with the rest). We generated scale-free networks using the Barabasi-Albert preferential attachment model (Barabasi and Albert, 1999). Figure $1 \mathrm{~A}$ (right, middle) shows a diagram of a scale-free network.

Small-world. The characteristic path length $L$ (i.e., the smallest number of edges separating any two vertices in the network, averaged over all such pairs) grows proportionally with the logarithm of the size of the network $n$ (i.e., $L \propto \log (n))$. This characteristic path length in small-world networks is significantly shorter compared with Erdős-Rényi networks with the same level of clustering. We generated small-world networks using a random rewiring procedure from the initial lattice network where each neuron connects to exactly 20 other neurons within the network (Watts and Strogatz, 1998). Figure $1 A$ (right, bottom) shows a diagram of a small-world network.

The connectivity from $\mathrm{Dbx} 1$ preBötC interneurons to reticular premotor neurons is also unknown. In the situations where the reticular network is modeled as scale-free or small-world, the preBötC is connected to the reticular network via an antipreferential attachment model. We computed a weighted connection probability $p_{i j}$ between preBötC neuron $i$ and reticular premotor neuron $j$ given by the following:

$$
p_{i j}=p_{\text {baseline }} * \frac{\text { average in-degree of all premotor neurons }}{\text { in-degree of premotor neurons } j}
$$

where $p_{\text {baseline }}$ is 0.175 .

\section{Numerical methods}

We simulated network models on the SciClone computing cluster at the College of William and Mary. We used a fourth-order Runge-Kutta numerical integration routine with a fixed time step of $0.25 \mathrm{~ms}$ using NeuronetExperimenter software (http://neuronetexp.sourceforge.net/) to simulate large populations of neurons with arbitrary connectivity. Network models were subjected to 100 random neuron deletions from either preBötC or reticular formation to measure the amplitude and frequency of respiratory-related network output, and then compare it with experimental results (Wang et al., 2014; Revill et al., 2015). One neuron was deleted every $20 \mathrm{~s}$ (simulated time) by setting the synaptic gating variable and its corresponding differential equation to zero, which disconnects the neuron from the rest of the network. Deleted neurons then no longer contributed to the fictive nerve output.

\section{Results}

Ablating preBötC and reticular interneurons cause disparate effects on respiratory rhythm and motor output

Cumulative photonic destruction of Dbx1 preBötC neurons impairs and then irreversibly precludes inspiratory rhythm generation (Wang et al., 2014). The amplitude of motor output (monitored from the XII cranial nerve root in vitro) decreased precipitously after ablating 10-15 Dbx1 neurons; further ablations decelerated spontaneous rhythm and then stopped it altogether after destruction of $\sim 85 \mathrm{Dbx} 1$ preBötC neurons. Figure $2 A$ shows a previously unpublished representative experiment from (Wang et al., 2014). Numerical simulations replicated perturbation and cessation of spontaneous rhythm when the model preBötC was subjected to cumulative random deletions (Song et al., 2015) but lacked the precipitous decrease in network output, which we postulate is because the model incorporated only the preBötC but not premotor or motor circuits.

Surmising that a more realistic model should feature reticular XII premotor circuits to properly replicate network output, we performed cell-selective photonic ablation experiments in rhyth- mically active slices to interrogate the role(s) of Dbx1 reticular neurons (Revill et al., 2015). The preBötC, reticular formation, and XII nuclei are bilaterally distributed in the brainstem, and that bilateral symmetry is retained in rhythmically active slices used for laser ablation experiments in vitro (for details, see Funk and Greer, 2013; Wang et al., 2014; Revill et al., 2015). Deleting Dbx1 reticular neurons from one side of the medullary slice preparation decreased the amplitude of the ipsilateral XII motor output linearly without affecting frequency. Figure $2 B$ shows a previously unpublished representative experiment from Revill et al., (2015). The contralateral XII output was unaffected. In general, selective laser ablation of $97 \pm 20$ (mean \pm SD) Dbx1 reticular neurons caused a $36 \pm 4 \%$ and $54 \pm 3 \%$ (mean \pm SD) decrease in ipsilateral XII amplitude and area, respectively (Revill et al., 2015).

\section{Erdős-Rényi network for the premotor population}

To attempt to replicate these in vitro experiments in silico, we first set up a network such that $36 \%$ of the preBötC neurons (i.e., 90 of the 250 model Dbx1 preBötC neurons) were allowed to connect to an Erdős-Rényi reticular network (e.g., Fig. 1A) with a fixed probability of 0.25 . Then we performed sequential random ablations from the model preBötC (Fig. 3).

The first model Dbxl preBötC neuron deletion was performed at $30 \mathrm{~s}$ (simulated time); a large portion of the XII premotor population started spiking tonically, which elevated the baseline of the running-time spike histogram. This running-time spike histogram encodes the firing patterns of all premotor neurons projecting to XII motoneurons; thus, it represents the model analog of XII nerve output recordings, which we dubbed "fictive nerve output" (see Figs. 3-5). The running-time spike histogram incorporates 50 preBötC neurons projecting to XII motoneurons (Fig. $1 A$, population $\mathrm{A}$ ) and the 100 reticular premotor neurons (Fig. $1 A$, population $\mathrm{D}$ ) and implicitly assumes that the XII motoneuron output is a linear readout of its constituent inputs. The tonic-spiking pattern in fictive nerve output (Fig. 3) was maintained throughout the simulation regardless of the ablation tally. Therefore, showing only the first four ablations suffices to demonstrate the outcome of the experiment. Here, the connectivity of the preBötC and reticular formation, as well as the connectivity within the reticular population itself, are independent. Reticular XII premotor neurons cannot generate rhythmic activity alone but rather entrain to rhythmic input from the preBötC. Therefore, as preBötC neurons are deleted, the reticular premotor neurons to which they project lose rhythmic synaptic drive but connectivity among reticular neurons remains unchanged. These premotor neurons, which have lost preBötC drive, cease to burst in sync with the preBötC but may engage in recurrent excitation with other reticular neurons and spike tonically.

Nine other simulations of the same network configuration showed qualitatively similar results (not shown). These data suggest that an Erdős-Rényi network is not a realistic model for the reticular premotor circuit because the outcome of in silico experiments (Fig. 3) did not match the corresponding in vitro experiments (Fig. 2A) (Wang et al., 2014), particularly with respect to the amplitude of motor output.

\section{Scale-free network for the XII premotor population}

Configuring the XII premotor population as a scale-free network (e.g., Fig. 1A) results in a large SD of the in-degree distribution (in-degree ranged from 22.9 to $77.0, \mathrm{SD}=14.2, N=50$ networks) compared with that of the Erdős-Rényi premotor popu- 
A

\section{preBötC Dbx1 neuron deletion}
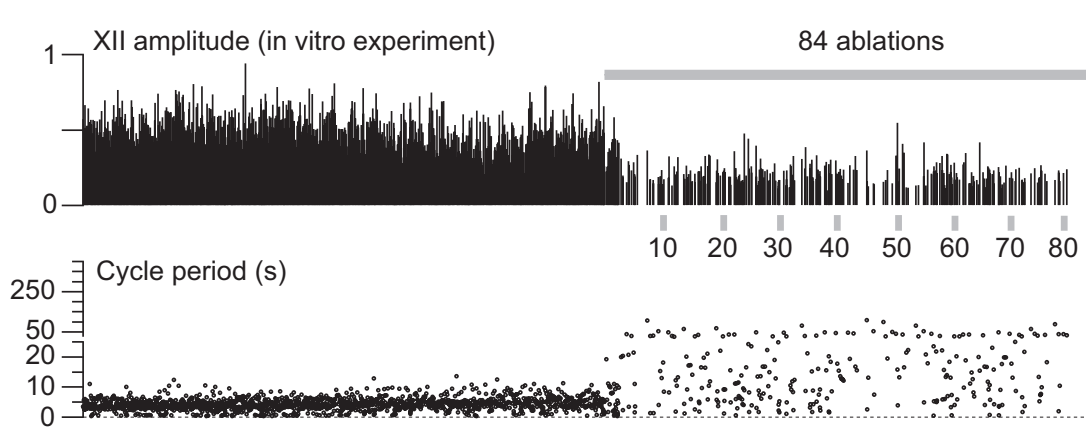

B

reticular premotor neuron deletion

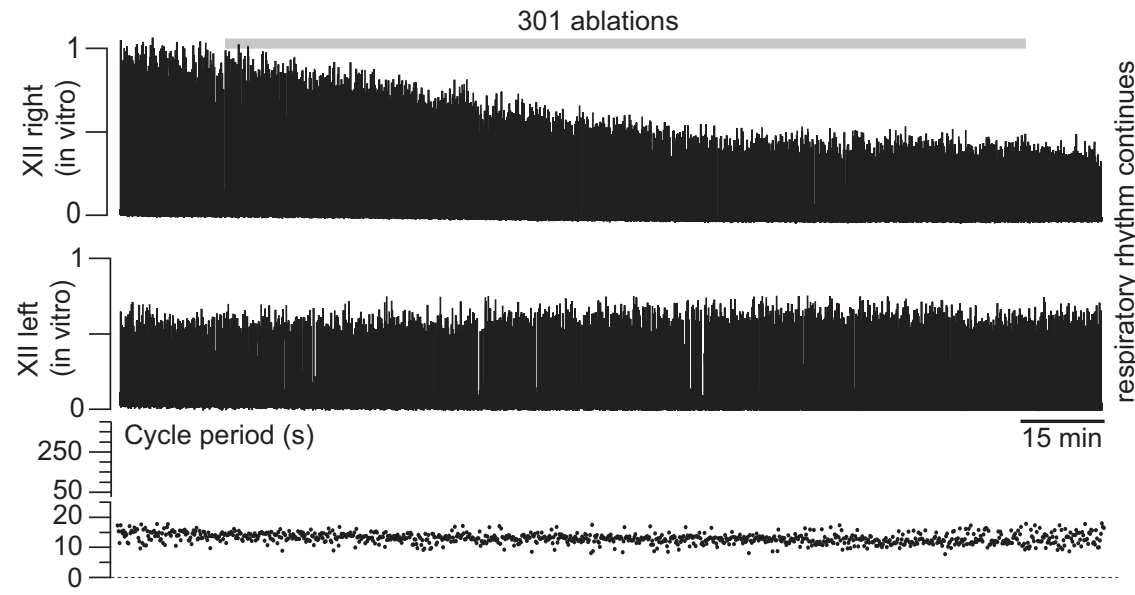

Figure 2. A, In vitro experiment showing that sequential, cumulative laser ablation of Dbx1 preBötC neurons induced precipitous decline in the inspiratory-related XII motor output, with concomitant rhythm deceleration followed by its cessation. Cellselective detection and laser-ablation experiments in the preBötC are thoroughly documented in the original publications (Hayes et al., 2012; Wang et al., 2013, 2014). In brief, slices that retain the preBötC, the intermediate reticular formation, as well as the XII motor nucleus and motor nerve root were perfused with $27^{\circ} \mathrm{C}$ artificial CSF at $4 \mathrm{ml} / \mathrm{min}$, and the external $\mathrm{K}^{+}$concentration was maintained at $9 \mathrm{~mm}$. Top, Normalized amplitude of the XII motor nerve discharge (motor output) measured via suction electrode and then amplified, full wave-rectified, and smoothed for display. Gray bar represents the cumulative laser-ablation phase with the total ablation tally. Bottom, Time-series plot of respiratory cycle period measured in real time during the laser-ablation experiment. The ordinate scale is continuous, but the tick scale changes at $25 \mathrm{~s}$ ( $5 \mathrm{~s}$ ticks are used from 0 to $25 \mathrm{~s} ; 50 \mathrm{~s}$ ticks are used from 26 to 400 s). Cycle period exceeding 250 s indicates irreversible rhythm termination. $B$, In vitro experiment showing sequential, cumulative Dbx1 reticular neuron laser ablations from the right side of the slice preparation in vitro, which caused a linear decline in the ipsilateral (right) XII amplitude with no concomitant perturbation of respiratory cycle period. Methodological details can be found in the original publication (Revill et al., 2015). Top, Normalized amplitude of the right XII motor nerve rootlet. Gray bar represents cumulative laser-ablation phase with the total ablation tally. Middle, Normalized amplitude measured from left XII nerve rootlet. Bottom, Time-series plot of respiratory cycle period measured in real time during the laser ablation experiment. To match $A$, the ordinate scale is continuous, but the tick scale changes at $25 \mathrm{~s}$ ( $5 \mathrm{~s}$ ticks are used from 0 to $25 \mathrm{~s} ; 50 \mathrm{~s}$ ticks are used from 26 to 400 s).

lation (in-degree ranged from 22.4 to $45.9, \mathrm{SD}=4.7, \mathrm{~N}=50$ networks). Nevertheless, high-in-degree vertices are sparse, so the overall network connectivity remained commensurate with the parameter sets identified in Figure $1 B$. Each premotor neuron in a scale-free reticular network received approximately the same number of synapses as in the Erdős-Rényi configuration (median in-degree measured 33.2 for the scale-free configuration and 33.5 for the Erdós-Rényi configuration).

We implemented an antipreferential attachment rule (see "Network model consisting of the preBötC and intermediate $\overline{10 \mathrm{~min}}$

reticular formation") when connecting preBötC neurons to the reticular premotor network (i.e., more interconnections with other reticular premotor neurons reduce the likelihood of input from the preBötC, and vice versa). Then, preBötC neurons were randomly and cumulatively ablated from the model system (Fig. 4A). Approximately half of the reticular premotor network stopped discharging bursts after the ninth deletion, which is qualitatively similar to the experimental results (Fig. 2A). Forty-one of 100 simulations of different realizations of the same network configuration showed qualitatively similar results (data not shown), in which $7.3 \pm 6.3$ (mean \pm $\mathrm{SD})$ neuron deletions would induce the precipitous amplitude decline, whereas $34.2 \pm 7.8$ (mean $\pm \mathrm{SD}$ ) neuron deletions led to rhythm termination. The other 59 simulations showed no qualitative change in the fictive nerve output as the growing ablation tally slowed and stopped the rhythm.

To further test the scale-free reticular network configuration, we randomly and cumulatively deleted XII premotor neurons matching the experiment in Figure $2 B(N=50)$. Fictive nerve output dropped initially and then stabilized (Fig. 4B) instead of decreasing linearly throughout the ablation sequence, which differed from the experimental result (Fig. $2 B)$. These data suggest that a scale-free network captures some, but not all, features of a realistic model for the reticular premotor circuit.

\section{Small-world network for the XII premotor population}

We sought to remodel the reticular premotor system while preserving connectivity commensurate with the ErdósRényi-like premotor network (to ensure synchronized activity in XII premotor neurons, see Fig. 1B) as well as the antipreferential attachment rule to connect the preBötC neurons with the reticular premotor network (which successfully replicated the amplitude drop in response to preBötC neuron ablation, see Figs. 2A, 4A).

Scale-free networks feature highly connected "hub" vertices whose deletion can cause abrupt changes in function, such as catastrophic system failure (Barabasi and Albert, 1999; Newman et al., 2001). We reasoned that hubs are unlikely to exist in the Dbx1 reticular premotor network because our cumulative ablation experiments in vitro never failed catastrophically (Fig. 2B) (Revill et al., 2015). If hubs exist in the real system, it seems unlikely that we would have failed to delete one in the context of hundreds of ablations randomly targeting Dbx1 reticular neurons performed in vitro. 

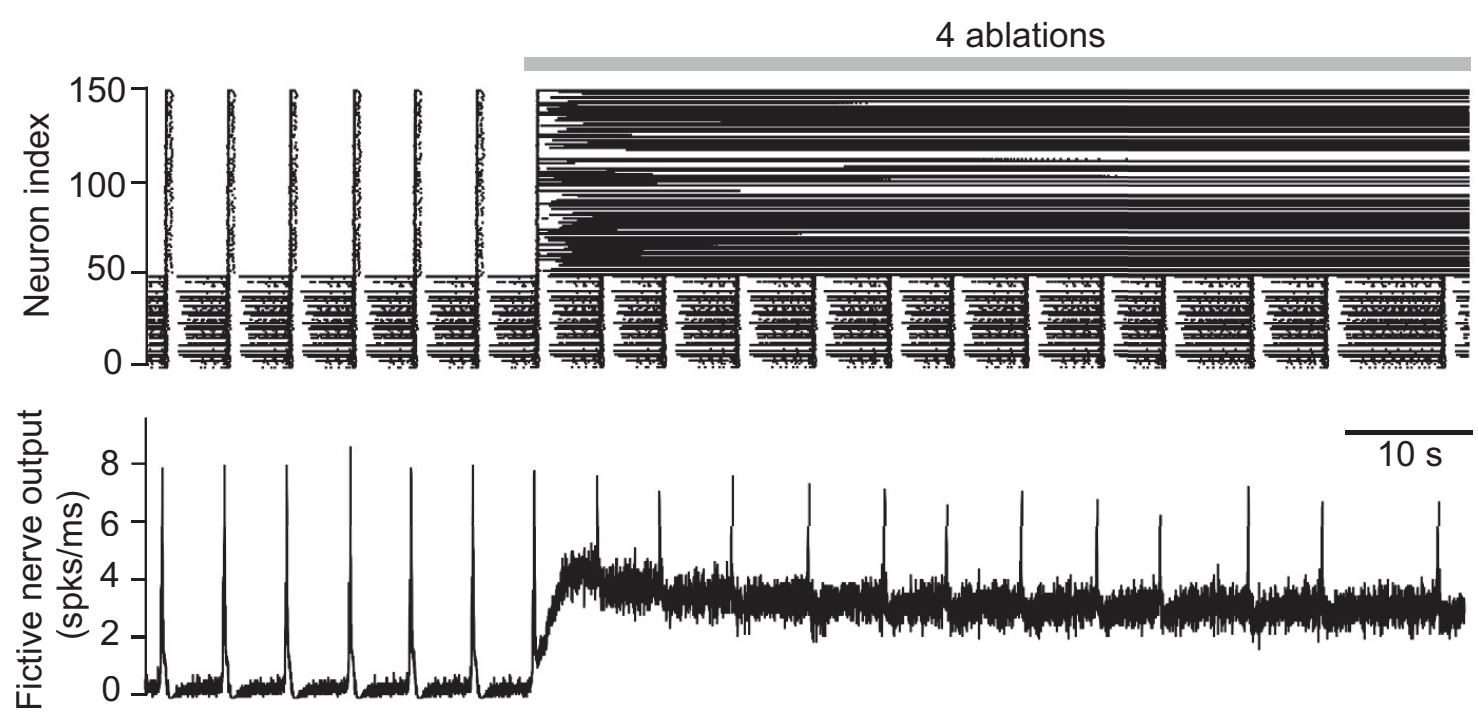

Figure 3. Simulated random deletion of preBötC neurons wherein the reticular premotor system is modeled as an Erdős-Rényi network. Top, Raster plot of 50 preBötC neurons (index 1-50) and 100 premotor neurons that project to the XII nucleus (index 51-150). Simulation lasts $100 \mathrm{~s}$ in total. Gray bar represents the cumulative laser-ablation phase with the total ablation tally. Each dot on the raster plot corresponds to a spike in a single neuron. Bottom, Running-time spike histogram (spks/ms) computed from the raster plot.

Therefore, we reassembled the reticular premotor system as a small-world network (e.g., Fig. 1A). The in-degree ranged from 28.3 to 40.3 ( $\mathrm{SD}=2.4$ ), and the median in-degree measured 34.0, which is similar to the in-degree distribution and the median in-degree of the Erdős-Rényi and scale-free network configurations. We again connected the preBötC to the reticular premotor network via antipreferential attachment.

We randomly deleted model Dbxl preBötC neurons and monitored fictive nerve output (Fig. 5A). The rhythm decelerated and terminated after a tally of 47 total ablations (the simulation reached a final tally of 53, but rhythm cessation occurred at 47); fictive nerve output experienced a precipitous drop in amplitude after the 13th deletion. These results broadly matched the experiments (Fig. 2A) (Wang et al., 2014). When we randomly deleted model Dbx1 neurons from the reticular premotor population instead, output decreased linearly to a steady-state level of attenuation without perturbing rhythmic frequency (Fig. 5B), which was also consistent with the Dbx1 reticular premotor neuron laser ablation experiments (Fig. 2B) (Revill et al., 2015).

In a total of 150 different realizations of the reticular premotor network in a small-world configuration, our simulations accurately reproduced the effects of preBötC neuron ablations in 38 cases $(25.3 \%$ of 150 simulations; $95 \%$ CI, 18\%-32\%), which is far from the majority of cases. However, the ability to accurately reproduce the reticular ablation experiment was much greater. In a total of 50 realizations of the reticular premotor network in a small-world configuration, we successfully replicated the results of Dbx1 reticular neuron ablations in 42 cases ( $84 \%$ of 50 simulations; $95 \%$ CI, 74\%-94\%). These data suggest the viability of a small-world network to model the real reticular premotor system but furthermore suggest that the precipitous drop in output following Dbx1 preBötC neuron ablation depends on additional factors that were not accurately captured in our simulations. Because the model preBötC neurons follow three qualitatively different coupling schemes (Fig. $1 A$, populations A-C), we speculated that a precipitous output drop, which invariably resulted from ablation experiments in vitro (Fig. 2A) (Wang et al., 2014), might depend on which certain subset of Dbx1 preBötC neurons are targeted for ablation.
To address this, we conducted three sets of preBötC neuron deletion simulations. Each set was repeated 50 times. Each run continued for 1030 simulated seconds (50 neuron deletions, one every $20 \mathrm{~s}$ after a $30 \mathrm{~s}$ initialization phase) and employed the same group of 50 network realizations referenced above. Only the random deletion sequence that targeted a specific subset of preBötC neurons changed in each case.

In the first case, we randomly deleted 50 neurons from among the 200 of the $\mathrm{Dbx} 1$ preBötC neurons that had no direct connection to the XII nucleus (sets $\mathrm{B}$ and $\mathrm{C}$ in Fig. 1A). For the second case, we specifically targeted the $50 \mathrm{Dbxl}$ preBötC neurons that connected to the XII nucleus (set A in Fig. 1A). For the third simulation, we selectively targeted 50 neurons from among the 90 Dbx1 preBötC neurons (set B in Fig. $1 A, 36 \%$ of 250 preBötC neurons) that connected to the reticular premotor population (set D in Fig. 1A). The fictive nerve output showed four characteristic patterns (Fig. 6) as follows: (1) lack of function, i.e., no network-wide bursts; (2) stable amplitude for the duration of the simulation; (3) tonic spiking in the premotor network after a certain number of ablations, causing a shift in the baseline amplitude (e.g., see Fig. 3 for a simulation with that type of output); and (4) precipitous amplitude drops after a number of neuron deletions (Figs. 2A, 4A, 5A) (Wang et al., 2014 for illustrations of this behavior).

Of the four patterns described above, only the fourth matches the in vitro targeted Dbx1 preBötC neuron laser-ablation experiments (Fig. 2A) (Wang et al., 2014). We categorized output patterns 1,2 , and 3 collectively as unsuccessful and output pattern 4 as successful. Five of 50 simulations (10\%) were successful in the first set of simulations, 16 simulations (32\%) were successful in the second group, and 42 simulations (84\%) were successful in the third group. These results indicate that the third strategy, which targeted Dbxl preBötC neurons that project to reticular premotor neurons (set B, Fig. $1 A$ ), caused a precipitous drop in the fictive nerve output amplitude more often than the other two strategies. Assuming the null hypothesis that all strategies would be equally effective, we performed a $\chi^{2}$ test to analyze the results $\left(\chi^{2}=59.28, p<0.00001\right)$. If targeting strategy has no impact on how the fictive nerve output responds to cumulative ablation, 
A

\section{preBötC Dbx1 neuron deletion}

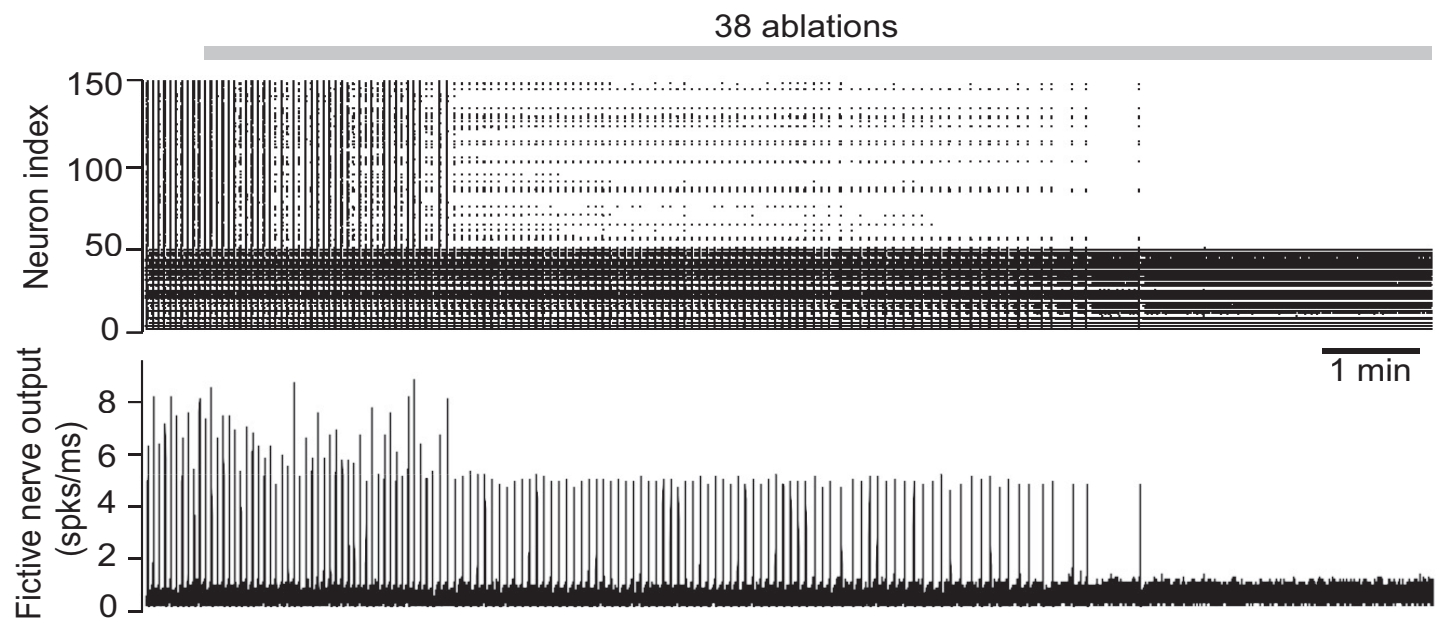

B

\section{reticular premotor neuron deletion}

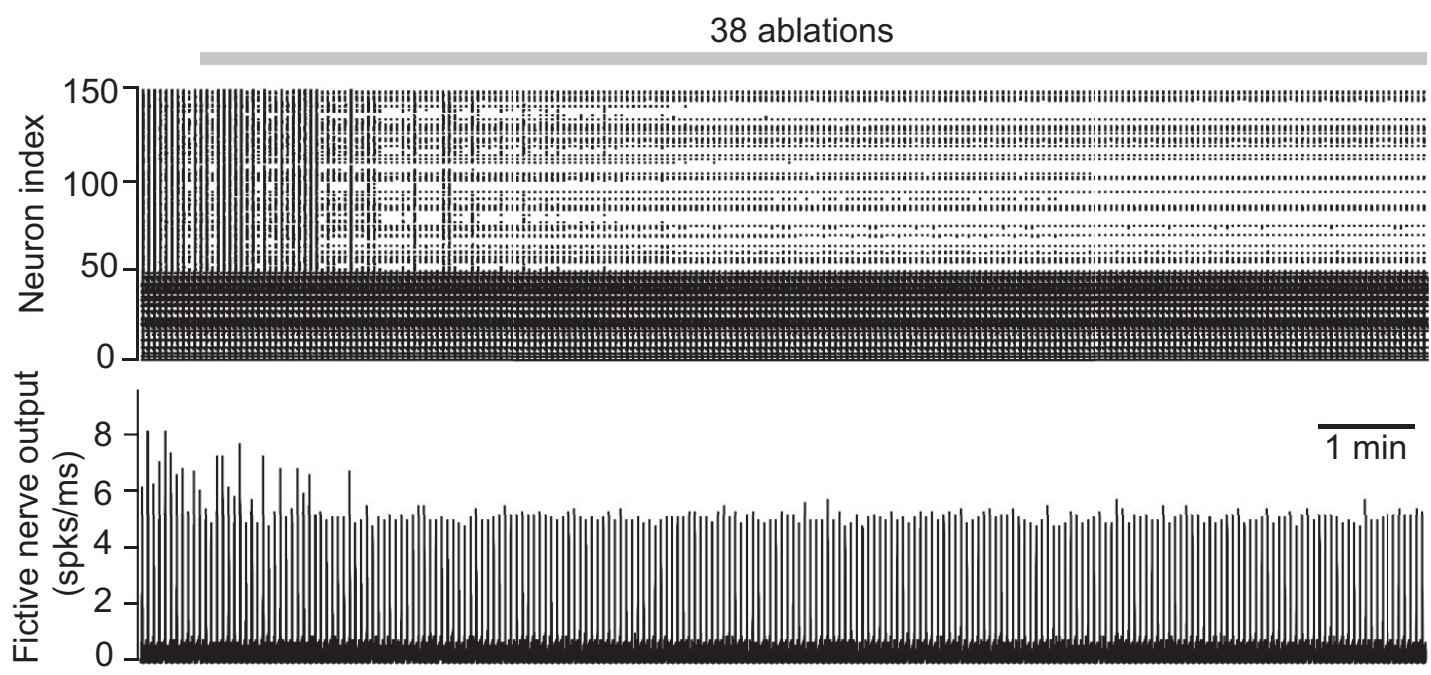

Figure 4. $\quad \boldsymbol{A}$, Simulated random deletion of preBötC neurons wherein the reticular premotor system is modeled as a scale-free network. $\boldsymbol{B}$, Simulated random deletion of reticular premotor neurons from a scale-free network configuration. Top, Raster plot of 50 preBötC neurons (index 1-50) and 100 premotor neurons that directly project to the XII nucleus (index 51-150). Simulation lasts $800 \mathrm{~s}$ in total. Gray bar represents the cumulative laser-ablation phase with the total ablation tally. Each dot on the raster plot corresponds to a spike in a single neuron. Bottom, Running-time spike histogram (spks/ms) computed from the raster plot.

then a result such as this would be extremely unlikely to occur by chance, which bolsters our confidence that targeting strategy is an important factor that influences the precipitous drop in network output characteristic of the in vitro laser ablation experiments.

\section{Discussion}

We developed laser ablation methodology with real-time physiological monitoring to quantify the cellular parameters of respiratory rhythm- and pattern-generating circuits (Hayes et al., 2012; Wang et al., 2013). Cumulative deletion of Dbx1 preBötC interneurons slowed and then stopped respiratory rhythm. Surprisingly, the magnitude of inspiratory motor output declined precipitously before the 15th neuron deletion. By contrast, laser ablation of Dbx1 reticular neurons decreased motor output linearly without affecting frequency. Here we present a plausible model that replicates these experiments and thus provides testable predictions regarding the configuration of rhythmogenic and XII premotor circuits.

The performance of this network system depends on the choice of the Rubin-Hayes model for preBötC and reticular premotor neurons. This model incorporates the two major burstgenerating inward charge carriers, $I_{\mathrm{Na}-\mathrm{P}}$ and $I_{\mathrm{CAN}}$, as well as excitatory synaptic connections. However, synaptically triggered intracellular $\mathrm{Ca}^{2+}$ dynamics coupled to $I_{\mathrm{CAN}}$ activation, which motivated the development of the Rubin-Hayes model, have 
A

preBötC Dbx1 neuron deletion

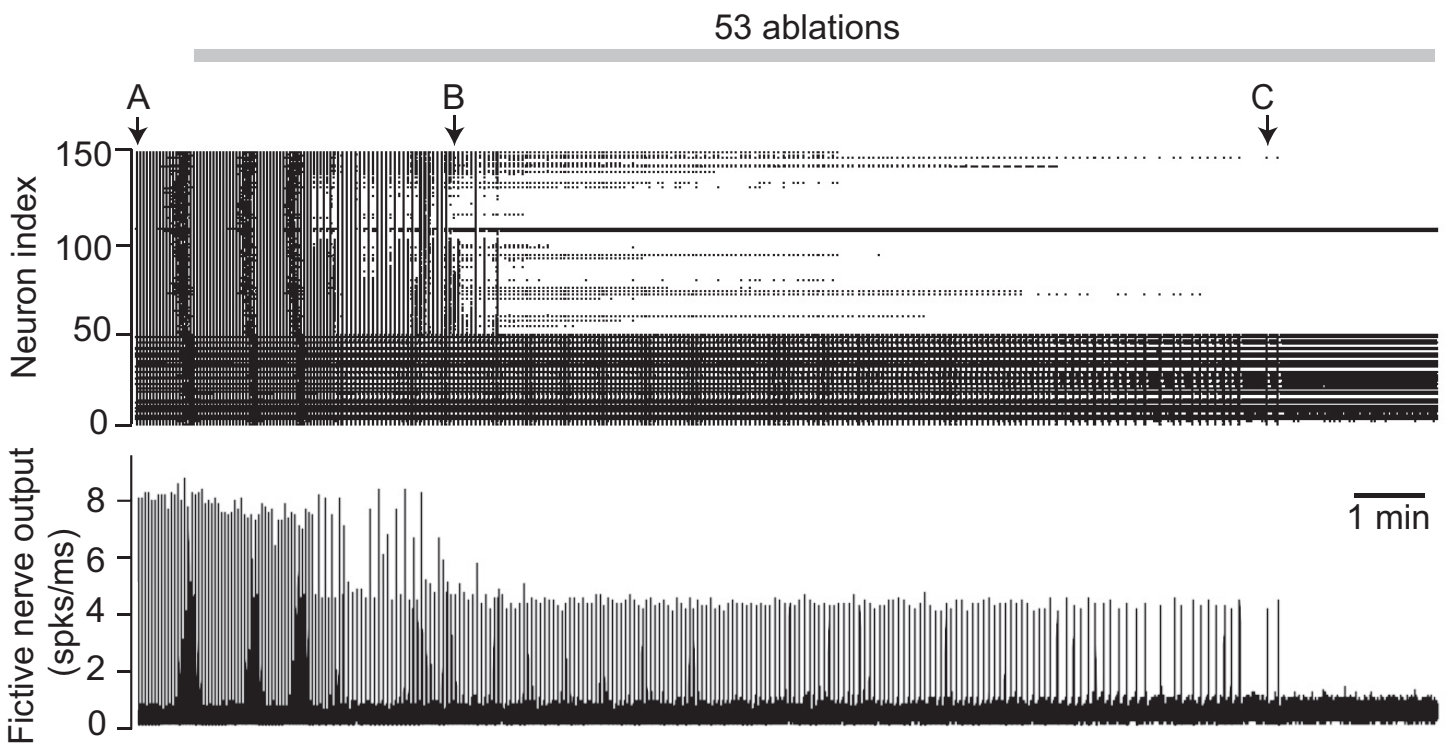

A

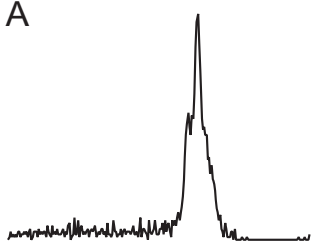

B

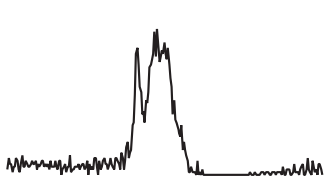

C

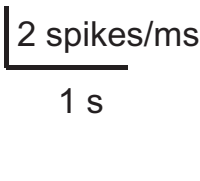

B

\section{reticular premotor neuron deletion}

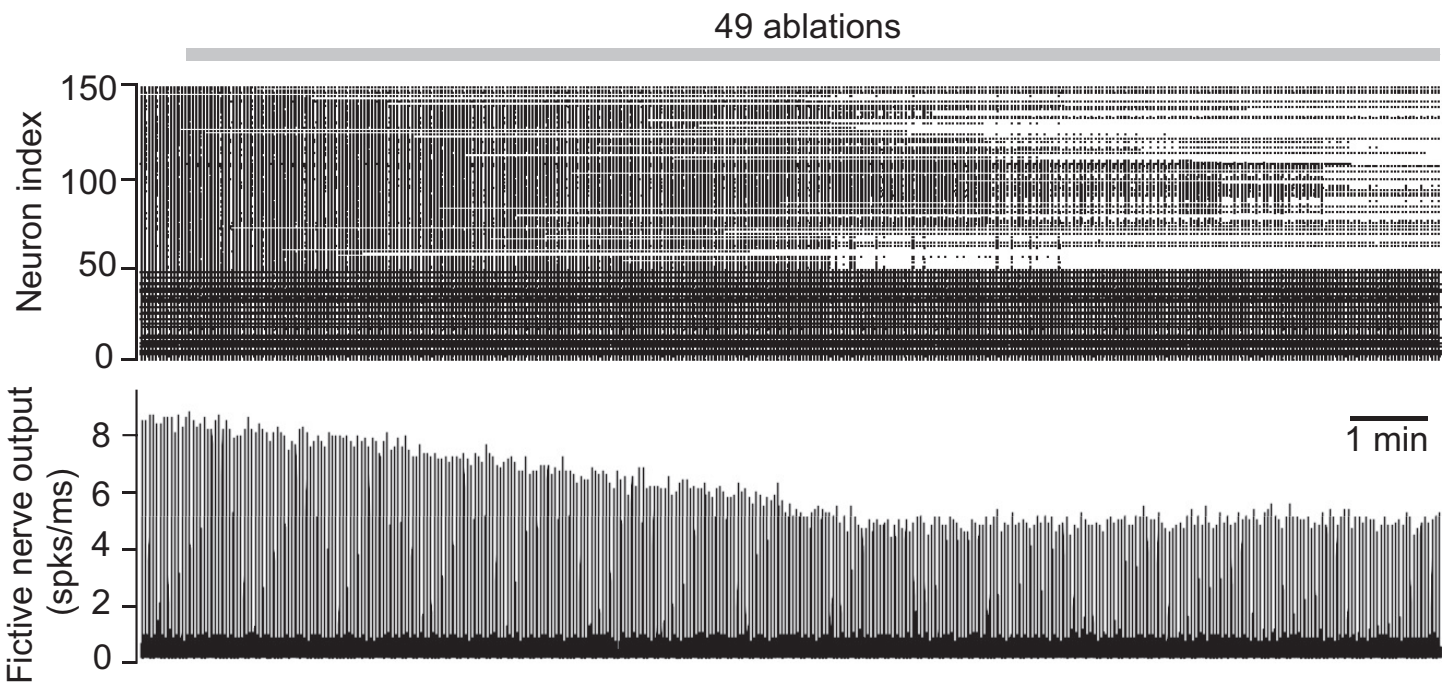

Figure 5. A, Simulated random deletion of preBötC neurons wherein the reticular premotor system is modeled as a small-world network with fictive nerve outputs for three individual network-wide bursts indicated by $\mathrm{A}-\mathrm{C}$ (indicated above the raster plot) at different stages of the simulated experiment. $\boldsymbol{B}$, Simulated random deletion of reticular premotor neurons from a small-world network configuration. Top, Raster plot of 50 preBötC neurons (index 1-50) and 100 premotor neurons that directly project to the XII nucleus (index 51-150). Simulation lasts 1000 s in total. Gray bar represents the cumulative laser-ablation phase with the total ablation tally. Each dot on the raster plot corresponds to a spike in a single neuron. Bottom ( $\boldsymbol{A}$, middle), Running-time spike histogram (spks/ms) computed from the raster plot. 


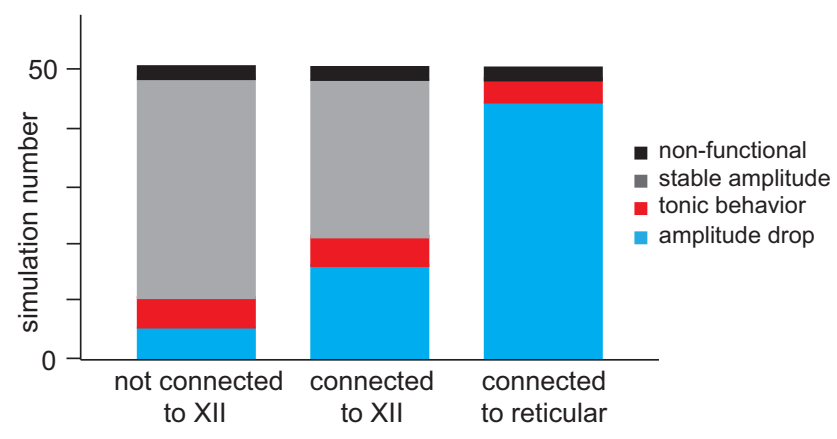

subset of preBötC neurons targeted for deletion

Figure 6. Deletion sequence analysis performed on 50 separate trials of cumulative ablation of model preBötC neurons. For three deletion strategies (horizontal axis), 50 simulations were conducted on the same group of 50 realizations of the same configuration for both the preBötC and a small-world reticular premotor network. Each simulation result falls into one of four possible outcomes, represented in different colors: black represents nonfunctional (i.e., the network never achieves a network-wide burst); gray represents stable amplitude (i.e., no precipitous decline in the output amplitude before rhythm termination); red represents tonic behavior (i.e., a large portion of premotor neurons spikes tonically after several preBötC neuron deletions); cyan represents precipitous amplitude drop (i.e., the rhythmic amplitude precipitously declined after a few neuron deletions).

been challenged by studies that perturbed metabotropic receptors and intracellular $\mathrm{Ca}^{2+}$ release without stopping rhythmogenesis (Beltran-Parrazal et al., 2012; Ben-Mabrouk et al., 2012). These results do not invalidate the model because Rubin-Hayes recapitulates recurrent excitation dynamics that all respiratory neurobiologists agree is essential for rhythmogesis (Funk et al., 1993; Wallén-Mackenzie et al., 2006; Carroll and Ramirez, 2013; Carroll et al., 2013); recurrent excitation also underlies another influential and contemporary model of respiratory rhythmogenesis (Guerrier et al., 2015). Furthermore, excitatory synapses evoke subthreshold $\mathrm{Ca}^{2+}$ accumulation in the Rubin-Hayes model via an agnostic mechanism not explicitly wedded to metabotropic receptors or intracellular $\mathrm{Ca}^{2+}$ release. Twophoton $\mathrm{Ca}^{2+}$ imaging in preBötC neurons dialyzed intracellularly with QX-314 demonstrate postsynaptic $\mathrm{Ca}^{2+}$ accumulation during inspiratory phases that does not depend on $\mathrm{Na}^{+}$spiking (Del Negro et al., 2011). Therefore, synaptically triggered subthreshold $\mathrm{Ca}^{2+}$ increases occur via an unknown mechanism whose dynamics are codified in the Rubin-Hayes model.

We have modeled the preBötC rhythmogenic core as an Erdős-Rényi network (Wang et al., 2014; Song et al., 2015). Several preBötC modeling studies used all-to-all connectivity (Butera et al., 1999; Purvis et al., 2007; Jasinski et al., 2013), which is not realistic: paired recordings in the preBötC showed that the excitatory synapses between putative rhythm generators are sparse ( $\sim 13 \%$ ) (Rekling et al., 2000). In contrast, Guerrier et al. (2015) constructed a random geometric network in which a synaptic connection from one neuron to another is exponentially proportional to the distance between them. The Guerrier model creates a far too robust preBötC whose rhythmic function stops after $44 \%$ of constituent neurons are deleted, whereas inspiratory rhythms in vitro and in our Erdős-Rényi model of the preBötC cease after $\sim 15 \%$ of the constituent neurons are ablated (Hayes et al., 2012; Wang et al., 2014; Song et al., 2015).

We created 100 reticular premotor neurons, initially coupled in Erdős-Rényi configuration, which showed that network-wide synchronization depends on $\sim 90$ of 250 preBötC neurons projecting to premotor neurons with a connection probability of $p=0.175$. This last parameter served as a baseline connection probability when we reconfigured the reticular premotor system as scalefree or small-world networks. Comparing laser ablation simulations to their corresponding experimental benchmarks provided testable insights into the structure and function of the real system.

\section{Scale-free and small-world reticular premotor networks and antipreferential attachment rule}

We compared three network configurations for the XII premotor population. The Erdős-Rényi premotor network synchronized well but did not replicate $\mathrm{Dbx} 1$ preBötC neuron ablation experiments. Deleting a model preBötC neuron diminishes rhythmic drive to the Erdős-Rényi premotor network, but its local connectivity has no relationship to preBötC input. So the premotor circuit may still undergo recurrent excitation, which leads to tonic spiking instead of rhythmic bursts in sync with the preBötC.

We surmised that connectivity between the preBötC and the reticular premotor system, and connectivity within the reticular population, cannot be independent. Alternative connectivity schemes should counterbalance synaptic input from reticular premotor neurons with synaptic drive from preBötC neurons.

We then modeled the XII premotor population as a scale-free network. The antipreferential attachment rule ensures that each XII premotor neuron receives proportional synaptic drive from both preBötC and premotor populations. It self-adjusts to maintain a fixed (total) level of synaptic input and is an important testable prediction for future studies.

Subsequently deleting model preBötC neurons predominantly affects XII premotor partners that receive fewer inputs from within the reticular network. Accordingly, XII premotor neurons that receive input from the preBötC are not connected richly enough within the reticular formation to promote recurrent excitation, which might otherwise lead to tonic spiking and induce the rest of the XII premotor population to spike tonically as well. By virtue of their relatively sparse connectivity within the reticular formation, these XII premotor neurons likely receive input from other remaining preBötC neurons and thus may continue to burst in sync with the preBötC, albeit with lower burst magnitude. Therefore, cumulatively deleting model preBötC neurons causes reticular premotor neurons to either burst with lower amplitude or go silent.

The scale-free premotor network configuration accurately replicated the Dbx1 preBötC neuron ablation experiments, but its output did not decline linearly in response to reticular neuron ablations, which occurred in every Dbx1 reticular neuron laserablation experiment.

We remodeled the XII premotor population as a small-world network, rich in local connections yet short in average path length, with antipreferential attachment connectivity from the preBötC. This configuration generated a precipitous decline in the fictive nerve output in the case of cumulative preBötC neuron deletions. Because each reticular premotor neuron in the smallworld network contributes commensurately to the network output, cumulatively deleting reticular neurons diminishes the fictive nerve output linearly as the ablation tally increases, consistent with the experiments (Revill et al., 2015). Our XII premotor population model comports with neuroanatomical evidence that some reticular formation networks are small-world (Achard et al., 2006; Bassett and Bullmore, 2006; Humphries et al., 2006).

Guerrier et al. (2015) demonstrated that synaptic dynamics (including facilitation and depression) can influence network behavior in ways that may override topological features. Could a disparity in synaptic dynamics, rather than network topology, 
explain the differential effects of laser ablation of Dbx1 preBötC versus Dbx1 reticular premotor neurons? Dbx1 preBötC neurons express synaptic properties predicted by Guerrier (Kottick and Del Negro, 2015), but similar information about excitatory synapses among Dbx1 reticular premotor neurons is not yet available. Nonetheless, Dbxl reticular premotor neurons have a lower level of excitability compared with Dbx1 preBötC neurons (Revill et al., 2015); thus, we modeled them as such. Without knowledge regarding synaptic disparity between preBötC and reticular premotor neurons, we favor a topological explanation for the differential effects of laser ablation.

\section{Implications for respiratory rhythm- and pattern-generating brainstem circuitry}

With a small-world reticular premotor network and antipreferential attachment from the preBötC, preBötC-neuron deletions caused a precipitous decline in the fictive nerve output in $\sim 25 \%$ of simulations (which occurred in $100 \%$ of the experiments). However, if we selectively targeted preBötC neurons directly connected to premotor neurons, then a precipitous decline in fictive nerve output occurred $84 \%$ of the time. Furthermore, when we examined all the simulations, we found that a precipitous decline in the fictive nerve output occurred by random chance whenever the deletion sequence contained a substantial fraction of preBötC neurons with direct connections to the reticular premotor system.

This analysis suggests that we preferentially photo-ablated Dbx1 preBötC neurons directly connected to the XII premotor network. The most parsimonious explanation would be that Dbx1 preBötC neurons that connect to reticular premotor neurons are concentrated at the rostral face of the preBötC, which is exposed by transverse slice preparations (Hayes et al., 2012; Wang et al., 2013, 2014). Laser ablation experiments would be predisposed to ablate these reticular-projecting neurons rather than other Dbx1 preBötC interneurons with only local connections. We cannot be sure that $\mathrm{Dbx} 1$ local-only preBötC interneurons are more prevalent at depth in the preBötC, but the evidence for XII premotor neurons at the rostral edge of the preBötC is strong. For example, premotor neurons for tongue protruder muscles are located in the area immediately rostral to and within the preBötC (Dobbins and Feldman, 1994). Dbxl neurons at the rostral-dorsal border of the preBötC project to the reticular formation (Picardo et al., 2013; Revill et al., 2015), and physiological transection and pharmacological studies attribute premotor function to the rostral edge of preBötC (Funk et al., 1993; Ruangkittisakul et al., 2014).

This study provides a framework for analysis of the respiratory rhythm- and pattern-generating circuits underlying breathing. An important prediction is the antipreferential connections from the preBötC to the small-world reticular formation. This could arise due to activity-dependent synaptic elimination (Flavell, 2006; Yogev and Shen, 2014) because an overabundance of synapses and neurons is commonplace during development (Cowan et al., 1984; O'Donovan, 1999). Analogous to our model assembly, the embryonic reticular formation may form a small-world network. Then, as preBötC synapses connect to reticular neurons, synapses are pruned to balance the excitation among reticular neurons and preBötC inputs during "fetal breathing" (Kobayashi et al., 2001). This effectively may account for how an antipreferential attachment rule emerges in the real system.

\section{References}

Achard S, Salvador R, Whitcher B, Suckling J, Bullmore E (2006) A resilient, low-frequency, small-world human brain functional network with highly connected association cortical hubs. J Neurosci 26:63-72. CrossRef Medline

Barabasi AL, Albert R (1999) Emergence of scaling in random networks. Science 286:509-512. CrossRef Medline

Bassett DS, Bullmore E (2006) Small-world brain networks. Neuroscientist 12:512-523. CrossRef Medline

Beltran-Parrazal L, Fernandez-Ruiz J, Toledo R, Manzo J, Morgado-Valle C (2012) Inhibition of endoplasmic reticulum $\mathrm{Ca}^{2+}$ ATPase in preBötzinger complex of neonatal rat does not affect respiratory rhythm generation. Neuroscience 224:116-124. CrossRef Medline

Ben-Mabrouk F, Amos LB, Tryba AK (2012) Metabotropic glutamate receptors (mGluR5) activate transient receptor potential canonical channels to improve the regularity of the respiratory rhythm generated by the pre-Bötzinger complex in mice. Eur J Neurosci 35:1725-1737. CrossRef Medline

Bouvier J, Thoby-Brisson M, Renier N, Dubreuil V, Ericson J, Champagnat J, Pierani A, Chédotal A, Fortin G (2010) Hindbrain interneurons and axon guidance signaling critical for breathing. Nat Neurosci 13:10661074. CrossRef Medline

Butera RJ Jr, Rinzel J, Smith JC (1999) Models of respiratory rhythm generation in the pre-Bötzinger complex: I. Bursting pacemaker neurons. J Neurophysiol 82:382-397. Medline

Carroll MS, Ramirez JM (2013) Cycle-by-cycle assembly of respiratory network activity is dynamic and stochastic. J Neurophysiol 109:296-305. CrossRef Medline

Carroll MS, Viemari JC, Ramirez JM (2013) Patterns of inspiratory phasedependent activity in the in vitro respiratory network. J Neurophysiol 109:285-295. CrossRef Medline

Chamberlin NL, Eikermann M, Fassbender P, White DP, Malhotra A (2007) Genioglossus premotoneurons and the negative pressure reflex in rats. J Physiol 579:515-526. CrossRef Medline

Cowan WM, Fawcett JW, O'Leary DD, Stanfield BB (1984) Regressive events in neurogenesis. Science 225:1258-1265. CrossRef Medline

Crowder EA, Saha MS, Pace RW, Zhang H, Prestwich GD, Del Negro CA (2007) Phosphatidylinositol 4,5-bisphosphate regulates inspiratory burst activity in the neonatal mouse preBötzinger complex. J Physiol 582:1047-1058. CrossRef Medline

Del Negro CA, Koshiya N, Butera RJ Jr, Smith JC (2002) Persistent sodium current, membrane properties and bursting behavior of pre-Bötzinger complex inspiratory neurons in vitro. J Neurophysiol 88:2242-2250. CrossRef Medline

Del Negro CA, Kam K, Hayes JA, Feldman JL (2009) Asymmetric control of inspiratory and expiratory phases by excitability in the respiratory network of neonatal mice in vitro. J Physiol 587:1217-1231. CrossRef Medline

Del Negro CA, Hayes JA, Rekling JC (2011) Dendritic calcium activity precedes inspiratory bursts in preBötzinger complex neurons. J Neurosci 31:1017-1022. CrossRef Medline

Dobbins EG, Feldman JL (1994) Brainstem network controlling descending drive to phrenic motoneurons in rat. J Comp Neurol 347:64-86. CrossRef Medline

Dunmyre JR, Del Negro CA, Rubin JE (2011) Interactions of persistent sodium and calcium-activated nonspecific cationic currents yield dynamically distinct bursting regimes in a model of respiratory neurons. J Comput Neurosci 31:305-328. CrossRef Medline

Feldman JL, Del Negro CA (2006) Looking for inspiration: new perspectives on respiratory rhythm. Nat Rev Neurosci 7:232-242. CrossRef Medline

Feldman JL, Del Negro CA, Gray PA (2013) Understanding the rhythm of breathing: so near, yet so far. Annu Rev Physiol 75:423-452. CrossRef Medline

Flavell SW, Cowan CW, Kim TK, Greer PL, Lin Y, Paradis S, Griffith EC, Hu LS, Chen C, Greenberg ME (2006) Activity-dependent regulation of MEF2 transcription factors suppresses excitatory synapse number. Science 311:1008-1012. CrossRef Medline

Fregosi RF, Bailey EF, Fuller DD (2011) Respiratory muscles and motoneurons. Respir Physiol Neurobiol 179:1-2. CrossRef Medline

Funk GD, Greer JJ (2013) The rhythmic, transverse medullary slice preparation in respiratory neurobiology: contributions and caveats. Respir Physiol Neurobiol 186:236-253. CrossRef Medline

Funk GD, Smith JC, Feldman JL (1993) Generation and transmission of respiratory oscillations in medullary slices: role of excitatory amino acids. J Neurophysiol 70:1497-1515. Medline

Ge Q, Feldman JL (1998) AMPA receptor activation and phosphatase inhi- 
bition affect neonatal rat respiratory rhythm generation. J Physiol 509: 255-266. CrossRef Medline

Gray PA, Hayes JA, Ling GY, Llona I, Tupal S, Picardo MC, Ross SE, Hirata T, Corbin JG, Eugenín J, Del Negro CA (2010) Developmental origin of preBötzinger complex respiratory neurons. J Neurosci 30:14883-14895. CrossRef Medline

Guerrier C, Hayes JA, Fortin G, Holcman D (2015) Robust network oscillations during mammalian respiratory rhythm generation driven by synaptic dynamics. Proc Natl Acad Sci U S A 112:9728-9733. CrossRef Medline

Hayes JA, Wang X, Del Negro CA (2012) Cumulative lesioning of respiratory interneurons disrupts and precludes motor rhythms in vitro. Proc Natl Acad Sci U S A 109:8286-8291. CrossRef Medline

Humphries M, Gurney K, Prescott T (2006) The brainstem reticular formation is a small-world, not scale-free, network. Proc Biol Sci 273:503-511. CrossRef Medline

Jasinski PE, Molkov YI, Shevtsova NA, Smith JC, Rybak IA (2013) Sodium and calcium mechanisms of rhythmic bursting in excitatory neural networks of the pre-Bötzinger complex: a computational modelling study. Eur J Neurosci 37:212-230. CrossRef Medline

Kobayashi K, Lemke RP, Greer JJ (2001) Ultrasound measurements of fetal breathing movements in the rat. J Appl Physiol 91:316-320. Medline

Koizumi H, Smith JC (2008) Persistent $\mathrm{Na}^{+}$and $\mathrm{K}^{+}$-dominated leak currents contribute to respiratory rhythm generation in the pre-Bötzinger complex in vitro. J Neurosci 28:1773-1785. CrossRef Medline

Koizumi H, Wilson CG, Wong S, Yamanishi T, Koshiya N, Smith JC (2008) Functional imaging, spatial reconstruction, and biophysical analysis of a respiratory motor circuit isolated in vitro. J Neurosci 28:2353-2365. CrossRef Medline

Koshiya N, Smith JC (1999) Neuronal pacemaker for breathing visualized in vitro. Nature 400:360-363. CrossRef Medline

Kottick A, Del Negro CA (2015) Synaptic depression influences inspiratoryexpiratory phase transition in Dbx1 interneurons of the preBötzinger complex in neonatal mice. J Neurosci 35:11606-11611. CrossRef Medline

Krey RA, Goodreau AM, Arnold TB, Del Negro CA (2010) Outward currents contributing to inspiratory burst termination in preBötzinger complex neurons of neonatal mice studied in vitro. Front Neural Circuits 4:124. CrossRef Medline

Li YX, Rinzel J (1994) Equations for InsP3 receptor-mediated $\left[\mathrm{Ca}^{2+}\right]$ i oscillations derived from a detailed kinetic model: a Hodgkin-Huxley like formalism. J Theor Biol 166:461-473. CrossRef Medline

Mironov SL (2008) Metabotropic glutamate receptors activate dendritic calcium waves and TRPM channels which drive rhythmic respiratory patterns in mice. J Physiol 586:2277-2291. CrossRef Medline

Mironov SL (2013) Calmodulin and calmodulin kinase II mediate emergent bursting activity in the brainstem respiratory network (preBötzinger complex). J Physiol 591:1613-1630. CrossRef Medline

Mironov SL, Skorova EY (2011) Stimulation of bursting in pre-Bötzinger neurons by Epac through calcium release and modulation of TRPM4 and K-ATP channels. J Neurochem 117:295-308. CrossRef Medline

Moore JD, Deschênes M, Furuta T, Huber D, Smear MC, Demers M, Kleinfeld D (2013) Hierarchy of orofacial rhythms revealed through whisking and breathing. Nature 497:205-210. CrossRef Medline

Newman ME, Strogatz SH, Watts DJ (2001) Random graphs with arbitrary degree distributions and their applications. Phys Rev E Stat Nonlin Soft Matter Phys 64:026118. CrossRef Medline

O’Donovan MJ (1999) The origin of spontaneous activity in developing networks of the vertebrate nervous system. Curr Opin Neurobiol 9:94104. CrossRef Medline

Ono T, Ishiwata Y, Inaba N, Kuroda T, Nakamura Y (1994) Hypoglossal premotor neurons with rhythmical inspiratory-related activity in the cat: localization and projection to the phrenic nucleus. Exp Brain Res 98:1-12. Medline

Pace RW, Del Negro CA (2008) AMPA and metabotropic glutamate receptors cooperatively generate inspiratory-like depolarization in mouse respiratory neurons in vitro. Eur J Neurosci 28:2434-2442. CrossRef Medline
Pace RW, Mackay DD, Feldman JL, Del Negro CA (2007) Inspiratory bursts in the preBötzinger complex depend on a calcium-activated non-specific cation current linked to glutamate receptors in neonatal mice. J Physiol 582:113-125. CrossRef Medline

Peever JH, Shen L, Duffin J (2002) Respiratory pre-motor control of hypoglossal motoneurons in the rat. Neuroscience 110:711-722. CrossRef Medline

Picardo MC, Weragalaarachchi KT, Akins VT, Del Negro CA (2013) Physiological and morphological properties of Dbx1-derived respiratory neurons in the pre-Bötzinger complex of neonatal mice. J Physiol 591: 2687-2703. CrossRef Medline

Ptak K, Zummo GG, Alheid GF, Tkatch T, Surmeier DJ, McCrimmon DR (2005) Sodium currents in medullary neurons isolated from the preBötzinger complex region. J Neurosci 25:5159-5170. CrossRef Medline

Purvis LK, Smith JC, Koizumi H, Butera RJ (2007) Intrinsic bursters increase the robustness of rhythm generation in an excitatory network. J Neurophysiol 97:1515-1526. CrossRef Medline

Rekling JC, Shao XM, Feldman JL (2000) Electrical coupling and excitatory synaptic transmission between rhythmogenic respiratory neurons in the preBötzinger complex. J Neurosci 20:RC113. Medline

Revill AL, Vann NC, Akins VT, Kottick A, Gray PA, Del Negro CA, Funk GD (2015) Dbx1 precursor cells are a source of inspiratory XII premotoneurons. eLife 4:e12301. CrossRef Medline

Ruangkittisakul A, Kottick A, Picardo MC, Ballanyi K, Del Negro CA (2014) Identification of the pre-Bötzinger complex inspiratory center in calibrated "sandwich" slices from newborn mice with fluorescent Dbx1 interneurons. Physiol Rep 2:e12111. CrossRef Medline

Rubin JE, Hayes JA, Mendenhall JL, Del Negro CA (2009) Calciumactivated nonspecific cation current and synaptic depression promote network-dependent burst oscillations. Proc Natl Acad Sci U S A 106: 2939-2944. CrossRef Medline

Smith JC, Ellenberger HH, Ballanyi K, Richter DW, Feldman JL (1991) PreBötzinger complex: a brainstem region that may generate respiratory rhythm in mammals. Science 254:726-729. CrossRef Medline

Song H, Hayes JA, Vann NC, Drew LaMar M, Del Negro CA (2015) Mechanisms leading to rhythm cessation in the respiratory preBötzinger complex due to piecewise cumulative neuronal deletions. eNeuro 2:ENEURO0031-15. 2015. CrossRef Medline

Stanek E 4th, Cheng S, Takatoh J, Han BX, Wang F (2014) Monosynaptic premotor circuit tracing reveals neural substrates for oro-motor coordination. eLife 3:e02511. CrossRef Medline

Volgin DV, Rukhadze I, Kubin L (2008) Hypoglossal premotor neurons of the intermediate medullary reticular region express cholinergic markers. J Appl Physiol 105:1576-1584. CrossRef Medline

Wallén-Mackenzie A, Gezelius H, Thoby-Brisson M, Nygård A, Enjin A, Fujiyama F, Fortin G, Kullander K (2006) Vesicular glutamate transporter 2 is required for central respiratory rhythm generation but not for locomotor central pattern generation. J Neurosci 26:12294-12307. CrossRef Medline

Wang X, Hayes JA, Picardo MC, Del Negro CA (2013) Automated cellspecific laser detection and ablation of neural circuits in neonatal brain tissue. J Physiol 591:2393-2401. CrossRef Medline

Wang X, Hayes JA, Revill AL, Song H, Kottick A, Vann NC, LaMar MD, Picardo MC, Akins VT, Funk GD, Del Negro CA (2014) Laser ablation of Dbxl neurons in the pre-Bötzinger complex stops inspiratory rhythm and impairs output in neonatal mice. eLife 3:e03427. CrossRef Medline

Watts DJ, Strogatz SH (1998) Collective dynamics of "small-world" networks. Nature 393:440-442. CrossRef Medline

Woch G, Ogawa H, Davies RO, Kubin L (2000) Behavior of hypoglossal inspiratory premotor neurons during the carbachol-induced, REM sleeplike suppression of upper airway motoneurons. Exp Brain Res 130:508520. CrossRef Medline

Yogev S, Shen K (2014) Cellular and molecular mechanisms of synaptic specificity. Annu Rev Cell Dev Biol 30:417-437. CrossRef Medline 